\title{
A Case of Squamous Cell Carcinoma of Unknown Primary that Responded to the Multi-Tyrosine Kinase Inhibitor Lenvatinib
}

\author{
Reiko Kimura-Tsuchiya ${ }^{a} \quad$ Eisaku Sasaki $^{a} \quad$ Izumi Nakamura $^{b}$ \\ Satoshi Suzuki ${ }^{\mathrm{b}}$ Satoshi Kawana ${ }^{c}$ Chiyo Okouchi ${ }^{\mathrm{b}}$ \\ Toshihiko Fukushima $^{b}$ Yuko Hashimoto ${ }^{c}$ Shinichi Suzuki ${ }^{b}$ \\ Shigehira Saji \\ ${ }^{a}$ Department of Medical Oncology, Fukushima Medical University, Fukushima, Japan; \\ ${ }^{b}$ Department of Thyroid and Endocrinology, Fukushima Medical University, \\ Fukushima, Japan; 'Department of Diagnostic Pathology, Fukushima Medical University, \\ Fukushima, Japan
}

\section{Keywords}

Carcinoma of unknown primary $\cdot$ Lenvatinib $\cdot$ Squamous cell carcinoma

\begin{abstract}
Lenvatinib is an oral tyrosine kinase inhibitor of vascular endothelial growth factor receptors 1,2 , and 3, fibroblast growth factor receptors 1 through 4, as well as platelet-derived growth factor receptor $\alpha$, RET, and KIT. At present, lenvatinib is used in the treatment of thyroid cancer and renal cell carcinoma. We herein report a case of a 67 -year-old patient with squamous cell carcinoma of unknown primary who was effectively treated with lenvatinib. The patient was initially diagnosed as having undifferentiated thyroid cancer, and after total thyroidectomy and bilateral lymph node dissection, lenvatinib was administered for the treatment of residual lymph node metastasis. A computed tomography scan after 1 month of lenvatinib administration showed marked regression of the lymph nodes, but interstitial pneumonia was also detected. Because the drug lymphocyte stimulation test for lenvatinib was strongly positive, we concluded that the interstitial pneumonia was induced by lenvatinib. The inter-
\end{abstract}


Kimura-Tsuchiya et al.: A Case of Squamous Cell Carcinoma of Unknown Primary that Responded to the Multi-Tyrosine Kinase Inhibitor Lenvatinib

stitial pneumonia only improved by the withdrawal of lenvatinib. Finally, his thyroid tumor was diagnosed as a metastasis of squamous cell carcinoma; however, we were unable to identify the primary lesion. This is the first reported case of interstitial pneumonia induced by lenvatinib.

\section{Introduction}

Lenvatinib is an oral, multi-targeted tyrosine kinase inhibitor of vascular endothelial growth factor receptors 1, 2, and 3, fibroblast growth factor receptors 1, 2, 3, and 4, plateletderived growth factor receptor $\alpha$, and RET and KIT signaling networks, which are involved in tumor growth [1-3]. Lenvatinib has been approved in 50 countries, including the US and the European Union, for the treatment of radioiodine-refractory differentiated thyroid cancer, and in Japan for the treatment of unresectable thyroid cancer $[4,5]$.

We herein report a case of squamous cell carcinoma of unknown primary that was effectively treated with lenvatinib.

\section{Case Presentation}

A 67-year-old man presented to a regional hospital with a tumor over the right clavicle. A computed tomography (CT) scan with contrast enhancement showed enlarged lymph nodes in the neck, mediastinum, and left axilla (Fig. 1a-c). A right supra-clavicular lymph node biopsy revealed poorly differentiated carcinoma. ${ }^{18} \mathrm{~F}$-fluorodeoxyglucose positron emission tomography/CT showed hot spots in the right lobe of the thyroid and lymph nodes (Fig. 1d). Although the tumor was unclear on CT and ultrasonography images, fine needle aspiration cytology of the right lobe of the thyroid showed poorly differentiated carcinoma cells similar to those in the observed lymph node. The serum level of the tumor marker carcinoembryonic antigen (CEA) was $1,390.0 \mathrm{ng} / \mathrm{mL}$ and the levels of the carbohydrate antigens 19-9 and 125 were 44.2 and $294 \mathrm{U} / \mathrm{mL}$, respectively. Based on a provisional diagnosis of undifferentiated thyroid cancer with lymph node metastasis, the patient underwent total thyroidectomy and bilateral lymph node dissection. Microscopically, undifferentiated carcinoma was suspected (Fig. 2). Lenvatinib ( $24 \mathrm{mg}$ /day) was administered for the treatment of residual lymph node metastasis. A CT scan after 1 month of lenvatinib administration showed marked regression of the lymph nodes in the mediastinum and left axilla; however, interstitial pneumonia was also detected in the bilateral lung (Fig. 3). The patient complained of mild cough and dyspnea on exertion, but had no fever. His oxygen saturation on room air was $98 \%$. Chest auscultation of both lung fields revealed no crackles, wheezes, or rhonchi, and chest X-ray showed bilateral infiltrative shadows (Fig. 3). His KL-6 (Krebs von den Lungen-6) levels $(<500 \mathrm{U} / \mathrm{mL})$ were $582 \mathrm{U} / \mathrm{mL}$, and his serum CEA levels decreased from 687 to $338 \mathrm{ng} / \mathrm{mL}$ after the administration of lenvatinib. The drug lymphocyte stimulation test for lenvatinib was strongly positive $(1,276 \mathrm{cpm}$, stimulation index 383 ; normal range: lower than 180). Broncho-alveolar lavage fluid revealed inflammatory changes with a cell differential count of $36.0 \%$ macrophages, $61.1 \%$ lymphocytes, $0 \%$ neutrophils, $2.9 \%$ eosinophils, and decreased CD 4/8 ratio (0.1). From these results, we diagnosed the patient as having lenvatinib-induced interstitial pneumonia. Lenvatinib was discontinued on the day of the CT scan (day 30). Due to the withdrawal, interstitial pneumonia improved remarkably. A pathologist who specializes in thyroid cancer diagnosed the patient's thyroid tumor as a 
Kimura-Tsuchiya et al.: A Case of Squamous Cell Carcinoma of Unknown Primary that Responded to the Multi-Tyrosine Kinase Inhibitor Lenvatinib

possible metastasis of poorly differentiated epithelial tumor. Further evaluation revealed that the tumor cells were positive for $\mathrm{p} 40$, demonstrating metastatic squamous cell carcinoma. Although the lung, head and neck were suspected as possible locations for the primary lesion, we were unable to identify the origin. During the 2-month period in which lenvatinib was not being administrated, the patient's CEA level increased, and a CT scan showed enlarged lymph nodes in the mediastinum and left axilla. He was treated for carcinoma of unknown primary with carboplatin and paclitaxel as second-line treatment. Following three cycles of chemotherapy with carboplatin and paclitaxel, stable disease was achieved in accordance with RECIST criteria (Fig. 4).

\section{Discussion}

There are two points of importance to be taken from this case: (1) lenvatinib was effective for squamous cell carcinoma and (2) to the best of our knowledge, this is the first report of interstitial pneumonia induced by administration of lenvatinib, which was confirmed by the drug lymphocyte stimulation test.

Lenvatinib is generally used to treat advanced and rapidly progressing thyroid cancer that can no longer be treated with radioactive iodine. Lenvatinib is also used with mammalian target of rapamycin inhibitor everolimus to treat advanced renal cell carcinoma as a second-line treatment. Lenvatinib for the treatment of hepatocellular carcinoma is in the process of approval in Japan, the US, Europe, and China.

In Europe and the US, lenvatinib is indicated for the treatment of locally recurrent or metastatic progressive, radioiodine-refractory differentiated thyroid cancer. In Japan, lenvatinib is not only used for differentiated but also for undifferentiated thyroid cancer. Therefore, in the current case, we administered lenvatinib for undifferentiated thyroid cancer that was finally diagnosed as squamous cell carcinoma of unknown primary. Currently, phase $\mathrm{Ib} / \mathrm{II}$ studies are being undertaken to investigate the efficacy and safety of lenvatinib in combination with pembrolizumab in some solid tumors (non-small cell lung cancer, endometrial cancer, urothelial cancer, head and neck cancer, and melanoma). The current report suggests that lenvatinib is also effective for some types of squamous cell carcinoma, probably because of tyrosine kinases targeted by this drug like vascular endothelial growth factor receptors, fibroblast growth factor receptors, platelet-derived growth factor receptor $\alpha$, KIT, and RET.

To date, new anticancer drugs, including molecular targeted drugs, have been developed and approved based on tumor origin. However, in May 2017, the US Food and Drug Administration approved treatment for patients whose cancers have specific biomarkers. In particular, pembrolizumab is indicated for the treatment of patients with unresectable or metastatic solid tumors that have been identified as having one of the following biomarkers: MSI$\mathrm{H}$ (microsatellite instability-high) or dMMR (deficient mismatch repair). Going forward, drugs should be developed and approved based on the tumor's biomarker, rather than its original location.

Among the clinical trials, no cases with interstitial pneumonia induced by lenvatinib have been reported. In an interim report of all cases of post-marketing surveillance (June 2017), this was the only case of interstitial pneumonia induced by lenvatinib.

Other tyrosine kinase inhibitors, such as gefitinib and erlotinib, are known to induce interstitial pneumonia, and as risk factors, performance status $2-4$, smoking history, and complications of interstitial lung disease were reported [6,7]. Our patient had performance status 0 , with a 15 pack-year smoking history and no complications of interstitial lung disease. 
Interstitial pneumonia was found on CT during follow-up observation, and was only improved by withdrawal of lenvatinib.

A limitation of this study was that comprehensive gene analysis of the tumor was not performed; therefore, it is not known whether an activated tyrosine kinase targeted by lenvatinib was present in squamous cell carcinoma in our patient.

\section{Conclusions}

The current report confirms the clinical effect of lenvatinib in a case with squamous cell carcinoma. In addition, to the best of our knowledge, this is the first case of interstitial pneumonia induced by lenvatinib.

\section{Statement of Ethics}

The authors have no ethical conflicts to declare.

\section{Disclosure Statement}

S. Saji has received honoraria and a research grant from Eisai Inc. The remaining authors declare that there are no conflicts of interest.

\section{References}

1 Okamoto K, Kodama K, Takase K, Sugi NH, Yamamoto Y, Iwata M et al. Antitumor activities of the targeted multi-tyrosine kinase inhibitor lenvatinib (E7080) against RET gene fusion-driven tumor models. Cancer Lett. 2013 Oct;340(1):97-103.

2 Matsui J, Funahashi Y, Uenaka T, Watanabe T, Tsuruoka A, Asada M. Multi-kinase inhibitor E7080 suppresses lymph node and lung metastases of human mammary breast tumor MDA-MB-231 via inhibition of vascular endothelial growth factor-receptor (VEGF-R) 2 and VEGF-R3 kinase. Clin Cancer Res. 2008 Sep;14(17): 5459-65.

3 Matsui J, Yamamoto Y, Funahashi Y, Tsuruoka A, Watanabe T, Wakabayashi T et al. E7080, a novel inhibitor that targets multiple kinases, has potent antitumor activities against stem cell factor producing human small cell lung cancer H146, based on angiogenesis inhibition. Int J Cancer. 2008 Feb;122(3):664-71.

4 Schlumberger M, Tahara M, Wirth LJ, Robinson B, Brose MS, Elisei R et al. Lenvatinib versus placebo in radioiodine-refractory thyroid cancer. N Engl J Med. 2015 Feb;372(7):621-30.

5 Kiyota N, Schlumberger M, Muro K, Ando Y, Takahashi S, Kawai Y et al. Subgroup analysis of Japanese patients in a phase 3 study of lenvatinib in radioiodine-refractory differentiated thyroid cancer. Cancer Sci. 2015 Dec;106(12):1714-21.

6 Murashige N, Tanimoto T, Oshima Y. Interstitial lung disease and gefitinib. N Engl J Med. 2010 Oct;363(16): 1578-9.

7 Inoue A, Saijo Y, Maemondo M, Gomi K, Tokue Y, Kimura Y et al. Severe acute interstitial pneumonia and gefitinib. Lancet. 2003 Jan;361(9352):137-9. 


\section{Case Reports in Oncology}

\begin{tabular}{l}
\hline Case Rep Oncol 2018;11:75-80 \\
\begin{tabular}{l|l}
\hline DOI: 10.1159/000486569 & $\begin{array}{l}\text { C } 2018 \text { The Author(s). Published by S. Karger AG, Basel } \\
\text { www.karger.com/cro }\end{array}$ \\
\hline
\end{tabular}
\end{tabular}

Kimura-Tsuchiya et al: A Case of Squamous Cell Carcinoma of Unknown Primary that Responded to the Multi-Tyrosine Kinase Inhibitor Lenvatinib
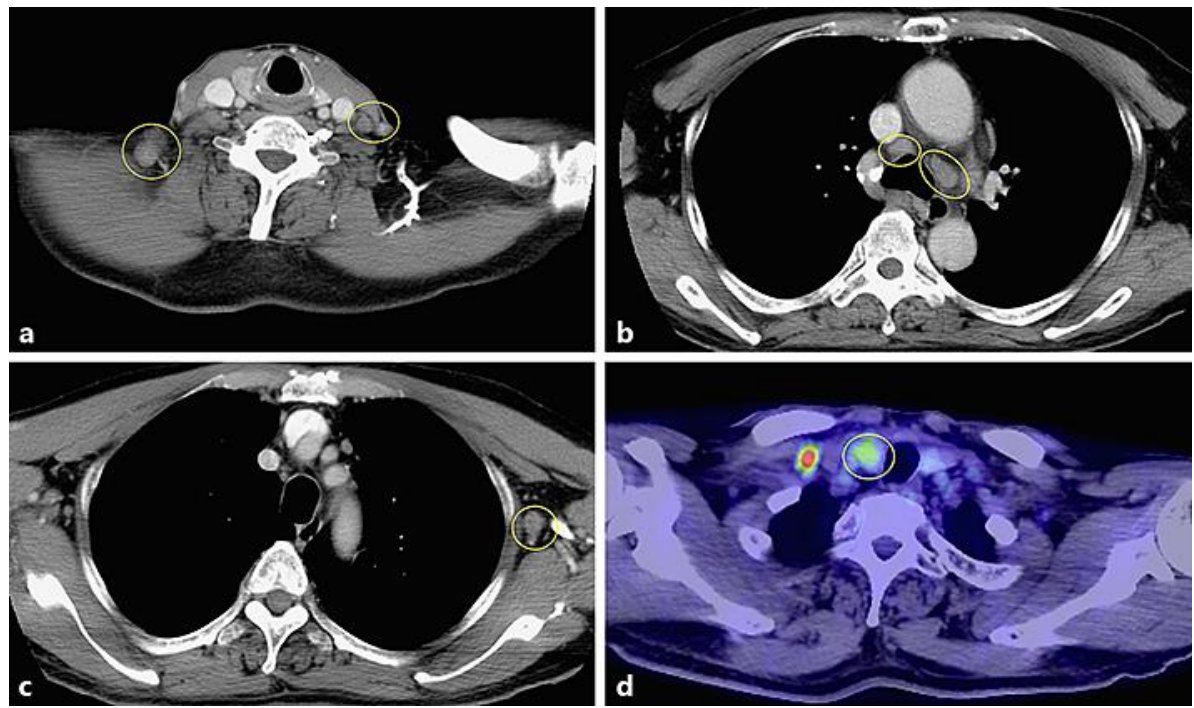

Fig. 1. Computed tomography (CT) scans with contrast enhancement (a-c) showed lymph node swelling of the neck, mediastinum, and left axilla. ${ }^{18} \mathrm{~F}$-fluorodeoxyglucose positron emission tomography/CT (d) showed a hot spot in the right lobe of the thyroid.
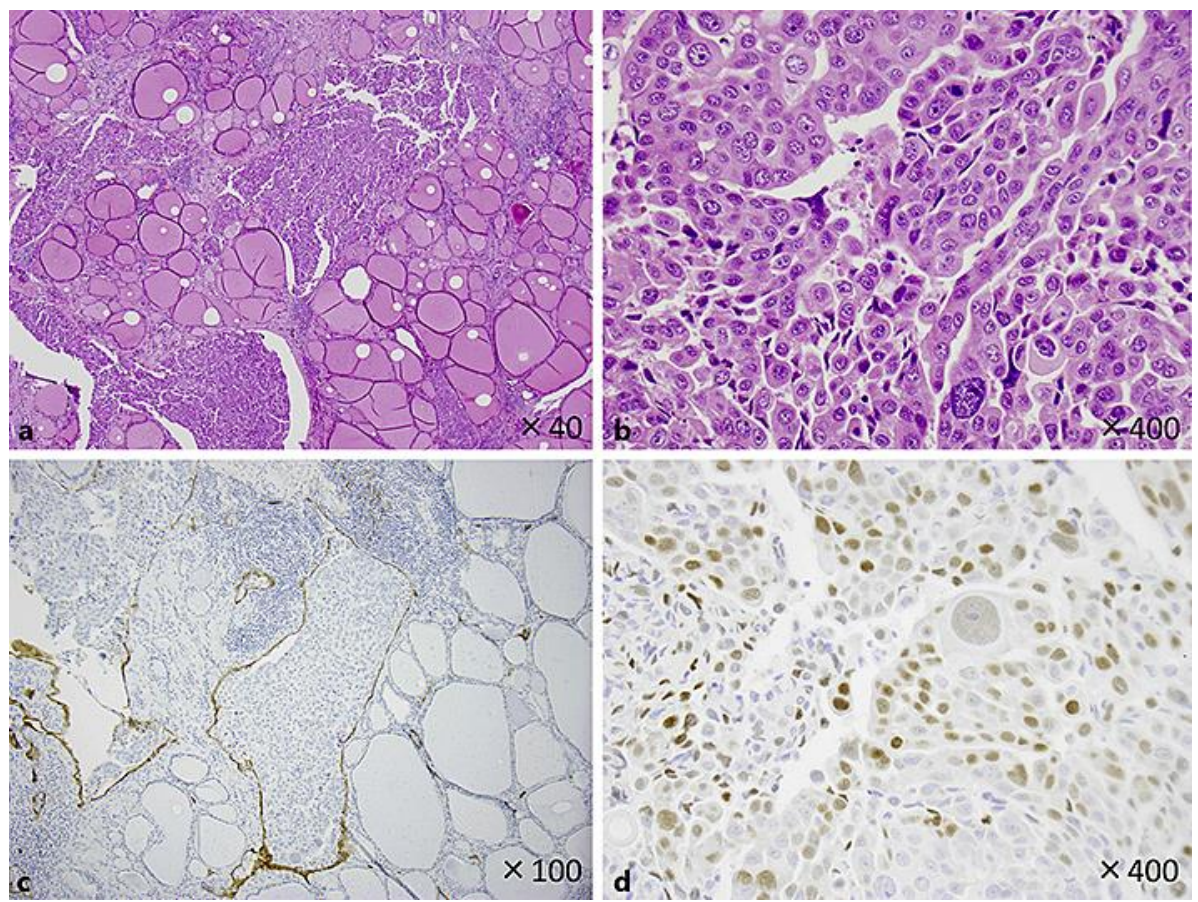

Fig. 2. Hematoxylin and eosin staining showed numerous foci of atypical cells between follicles in the thyroid tissue. The tumor cells in $\mathbf{a}$ and $\mathbf{b}$ showed sheet-like arrangement, but no keratinization, intercellular bridge, glandular structure or mucin production. Because of such undifferentiated features, the tumor was diagnosed as undifferentiated thyroid cancer at first. Further study revealed that all tumor cells were within the D2-40-positive lymph ducts, did not infiltrate thyroid parenchyma (c), and were positive for p40 (d). These results showed that the tumor is metastatic squamous cell carcinoma of unknown primary. 


\section{Case Reports in Oncology}
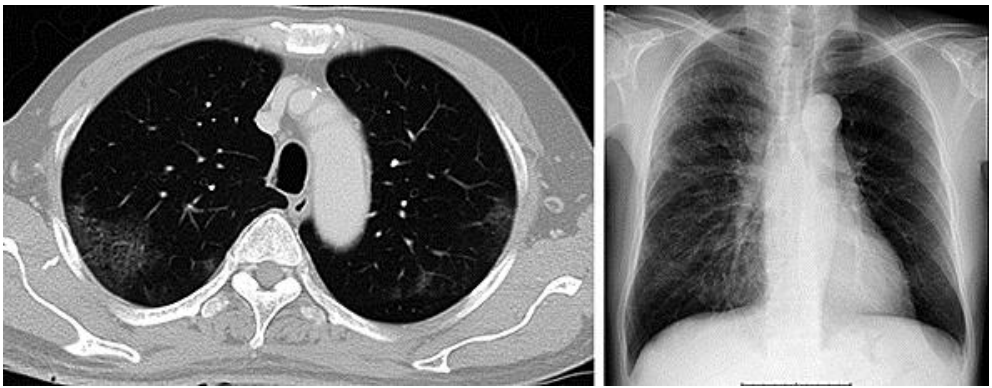

Fig. 3. A chest CT scan showed mosaic patterns with ground-glass opacities in both lungs (left). A chest X-ray taken on day 35. Diffuse infiltrative shadows were observed in both lung fields (right).

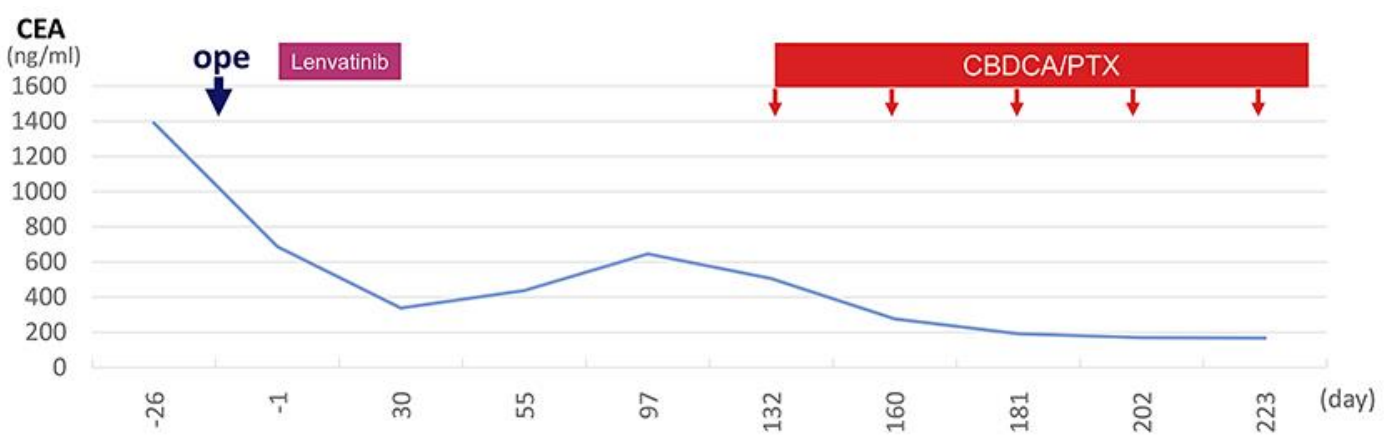

Fig. 4. Clinical course of the patient. Day 1 is the starting date of the first cycle of lenvatinib administration. CBDCA, carboplatin; PTX, paclitaxel. 